\title{
Effect of membrane polymeric materials on relationship between surface pore size and membrane fouling in membrane bioreactors
}

Taro Miyoshi ${ }^{1}$, Kotaku Yuasa $^{l}$, Toru Ishigami ${ }^{1}$, Saeid Rajabzadeh $^{l}$, Eiji Kamio ${ }^{1}$, Yoshikage Ohmukai ${ }^{1}$, Daisuke Saeki ${ }^{l}$, Jineren $\mathrm{Ni}^{2}$, Hideto Matsuyama ${ }^{1 *}$

${ }^{1}$ Center for Membrane and Film Technology, Department of Chemical Science and Engineering, Kobe University, 1-1 Rokkodai, Nada-ku, Kobe 657-8501, Japan

${ }^{2}$ Department of Environmental Engineering, Peking University; The Key Laboratory of Water and Sediment Sciences, Ministry of Education, Beijing 100871, China

Taro Miyoshi: E-mail address: t-miyoshi@ pegasus.kobe-u.ac.jp

Kotaku Yuasa: E-mail address: k.yuasa06@gmail.com

Toru Ishigami: E-mail address: ishigami.toru@ @ihon-u.ac.jp

Eiji Kamio: E-mail address: e-kamio@ people.kobe-u.ac.jp

Yoshikage Ohmukai: E-mail address: yoshikage.ohmukai@ daikin.co.jp

Daisuke Saeki: E-mail address: dsk.saeki@garnet.kobe-u.ac.jp

Jineren Ni: E-mail address: jinrenni@pku.edu.cn

* Corresponding author, Hideto Matsuyama: Tel \& Fax: +81-78-803-6180, E-mail: matuyama@kobe-u.ac.jp 


\begin{abstract}
We investigated the effect of different membrane polymeric materials on the relationship between membrane pore size and development of membrane fouling in a membrane bioreactor (MBR). Membranes with different pore sizes were prepared using three different polymeric materials, cellulose acetate butyrate $(\mathrm{CAB})$, polyvinyl butyral (PVB), and polyvinylidene fluoride (PVDF), and the development of membrane fouling in each membrane was evaluated by batch filtration tests using a mixed liquor suspension obtained from a laboratory-scale MBR. The results revealed that the optimal membrane pore size to mitigate membrane fouling differed depending on membrane polymeric material. For PVDF membranes, the degree of membrane fouling decreased as membrane pore size increased. In contrast, $\mathrm{CAB}$ membranes with smaller pores had less fouling propensity than those with larger ones. Such difference can be attributed to the difference in major membrane foulants in each membrane; in PVDF, they were small colloids or dissolved organics in which proteins are abundant, and in $\mathrm{CAB}$, microbial flocs. The results obtained in this study strongly suggested that optimum operating conditions of MBRs differ depending on the characteristics of the used membrane.
\end{abstract}

Keywords: Membrane fouling, Membrane polymeric materials, Pore size, Membrane bioreactor (MBR), Polyvinylidene fluoride (PVDF) 


\section{Introduction}

Membrane bioreactors (MBRs) have many advantages over conventional wastewater treatment technologies, including high quality of treated water, small footprint, and ease of operation [1,2], and are becoming increasingly popular for wastewater treatment [3]. However, high operation and maintenance costs that can mainly be attributed to the development of membrane fouling (deterioration of membrane permeability) are obstacles that limit the widespread application of this technology. To use MBRs more efficiently, strategies to control membrane fouling need to be established.

Membrane fouling in MBRs has therefore been a hot research topic and numerous papers on this subject have been published [4,5]. Attention has been paid to various design and operating parameters such as membrane flux [6-10], solid retention time (SRT) [11-13], and food-to-microorganism (F/M) ratio [14-16]. In addition to these factors, it is widely accepted that the membranes used also greatly influence the development of membrane fouling in MBRs [17-22]. These previous studies suggested that the development of membrane fouling in MBRs could be substantially suppressed by using an appropriate membrane. At present, however, a clear consensus on the characteristics of membranes with low fouling propensity has not been established.

Generally, membranes with smooth surface morphology are thought to be desirable for preventing membrane fouling [18, 20, 21]. However, Choi et al. [19] reported that pore structure has a greater influence on the development of membrane fouling than the roughness of membrane surfaces. The effect of pore size on the development of membrane fouling is also complicated. While some researchers reported that membranes with larger pore sizes are more susceptible to membrane fouling [20], 
the opposite result has also been published [22]. The effect of pore size on membrane fouling strongly depends on the pure water permeability (PWP) of a membrane. Membranes with higher PWP are generally believed to have lower fouling propensity $[18,19,21]$. It is still open for discussion whether the hydrophobicity of a membrane affects the development of membrane fouling or not. Although van der Marel et al. [22] reported that hydrophobic membrane was fouled more severely than hydrophilic one by comparison of polyvinyl fluoride (PVDF) membranes with different hydrophobicity (other membrane properties were basically the same), other researchers have reported that the effect of membrane hydrophobicity on the development of membrane fouling was not significant compared with other indexes representing membrane properties $[18,19,21]$. Hydrophilic membranes may be capable of mitigating the development of membrane fouling to some extent, but the influence of membrane hydrophobicity on the development of membrane fouling is likely to be less important than those of other membrane properties such as PWP or pore structure.

As summarized above, many contradictory results regarding the properties of membranes that affect fouling formation have been reported. One possible reason for these discrepancies might be the difficulty in controlling membrane properties to solely evaluate the effect of a particular membrane property on the development of membrane fouling. For example, membranes with high porosity tend to have high PWP. This means that sufficient information cannot be obtained when small number of membranes are examined. On this basis, here we comprehensively investigated which of membrane properties affect on the development of membrane fouling in MBRs. Membranes with different pore sizes were prepared using three different membrane polymeric materials, cellulose acetate butyrate (CAB), polyvinyl butyral (PVB), and PVDF, and the 
development of membrane fouling in each membrane was evaluated by batch filtration testing using mixed liquor suspensions obtained from a laboratory-scale MBR. On the basis of the results obtained in this study, the effect of membrane pore size on the development of membrane fouling caused by the difference in membrane polymeric materials was discussed.

\section{Materials and methods}

\subsection{Materials}

PVDF (Mw = 322,000, Solvay Specialty Polymers Japan K.K., Tokyo, Japan), PVB $(\mathrm{Mw}=135,000$, Denki Kagaku Kogyo K.K., Tokyo, Japan $)$, and CAB $(\mathrm{Mw}=$ 65,000, Daicel Chemical Industries, Osaka, Japan) were used as membrane polymeric materials. Diethyl phthalate (DEP, Wako Pure Chemical Industries, Osaka, Japan), triethylene glycol (TEG, Nacalai Tesque Inc., Kyoto, Japan), and polyethylene glycol 200 (PEG 200, Wako Pure Chemical Industries) were used as diluents for membrane preparation via thermally induced phase separation (TIPS). A Pierce BCA Protein Assay kit was purchased from Thermo Scientific. All chemicals were used without further purification.

\subsection{Membrane preparation}

All hollow fiber membranes used in this study were prepared using a batch-type extruder (BA-0, Imoto Co., Kyoto, Japan) by TIPS [23]. The preparation conditions are summarized in Table 1. Membranes with different pore sizes were prepared by changing the polymer concentration and air gap. Detailed protocols of membrane preparation have been reported elsewhere [24]. 
To evaluate the pore size of the prepared membranes, filtration experiments were carried out. Solution of monodisperse polystyrene latex particles with diameters of 20, 50, 100 and $300 \mathrm{~nm}$ (Thermo Fisher Scientific, MA, USA) containing $0.1 \mathrm{wt} \%$ Triton X-100 (Wako Pure Chemical Industries) were used as the feed solution. The particle rejection was determined by measuring the solute concentration in the feed and filtrate solutions using an ultraviolet-visible spectrophotometer (U-200, Hitachi, Ltd., Tokyo, Japan) to measure absorption at $380 \mathrm{~nm}$. We used the particle diameter when the rejection was $90 \%$ as the pore size of each membrane. Refer to our previous report for details [25]. Although not shown here, observation of the pores for membranes with small pore size and recognition of the difference in pore size for diferent membranes based on the SEM images was difficult and not accurate enough. Thus, for current study membranes pore size was determined by polystyrene latex particles filtration experiment.

To evaluate the hydrophobicity of the prepared membranes, the water contact angle of the film surface was measured using a contact angle meter (DM-300; Kyowa Interface Science, Saitama, Japan).

\subsection{Operation of a laboratory-scale MBR}

A laboratory-scale MBR was operated continuously. Two hollow fiber membrane modules where the effective membrane surface area of each module was $0.015 \mathrm{~m}^{2}$ were simultaneously submerged in the same MBR tank $(10 \mathrm{~L})$ and filtered the same mixed liquor suspension side by side. The MBR tank was initially filled by the wastewater supplied from industrial wastewater treatment plant at Shizuka prefecture, Japan. These membrane modules were operated under a constant membrane flux of 0.23 
$\mathrm{m}^{3} / \mathrm{m}^{2} /$ day $\left(9.6 \mathrm{~L} / \mathrm{m}^{2} / \mathrm{h}\right)$. Hydraulic retention time (HRT) and SRT were set at $35 \mathrm{~h}$ and 90 days, respectively. In this study, two separate continuous operations (Runs 1 and 2) were carried out. In Run 1, the MBR was fed with synthetic wastewater composed of $525 \mathrm{mg} / \mathrm{L}$ skimmed milk and $175 \mathrm{mg} / \mathrm{L}$ bonito extract (Kyokuto Pharmaceutical Industrial, Japan). The composition of synthetic wastewater used in Run 2 was $1.37 \mathrm{~g} / \mathrm{L}$ sucrose, $0.27 \mathrm{~g} / \mathrm{L}$ acetic acid, $0.32 \mathrm{~g} / \mathrm{L}$ ammonium sulfate, and $0.062 \mathrm{~g} / \mathrm{L}$ disodium hydrogen phosphate. The mixed-liquor suspended solids (MLSS) concentration was approximately $15 \mathrm{~g} / \mathrm{L}$ in both runs.

\subsection{Batch filtration test using micro-membrane modules}

A series of batch filtration experiments was carried out to evaluate the effect of membrane properties on fouling propensity. A schematic diagram of the experimental apparatus is shown in Fig. 1. Micro-membrane modules with a membrane fiber length of approximately $7 \mathrm{~cm}$ were prepared using the membranes fabricated in this study. New membrane fibers were used in each test. Each micro-membrane module was installed in a small filtration apparatus with an effective volume of approximately 120 $\mathrm{mL}$. The inside of the filtration apparatus was filled with mixed liquor suspension obtained from the laboratory-scale MBR in Run 2 described above, and filtration was carried out with a constant membrane flux of $0.23 \mathrm{~m}^{3} / \mathrm{m}^{2} /$ day (the same membrane flux as used in the continuous operation of the laboratory-scale MBR). To avoid concentrating the mixed liquor suspension in the filtration apparatus, $0.85 \mathrm{wt} \% \mathrm{NaCl}$ aqueous solution was supplied to compensate for the loss of mixed liquor suspension by membrane filtration.

The batch filtration tests were conducted using two different feed suspensions: 
whole mixed liquor suspension without any treatment and the supernatant obtained following centrifugation at $8000 \times$ g for 10 min (himac CF15RX II, Hitachi Koki Co., Tokyo, Japan). By visual inspection, supernatants were clear, suggesting that no suspended solids remained after centrifugation described above. The batch filtration experiments using the supernatant from centrifugation should represent the membrane fouling caused by dissolved matter and small organic colloids in mixed liquor suspension.

To estimate the effect of protein concentration on the development of membrane fouling, the batch filtration tests were also performed using the supernatant from mixed liquor suspensions with different protein concentrations. Proteins were selectively removed by filtering the supernatant through a PVC copolymer membrane with nominal pore size of $0.8 \mu \mathrm{m}$ (PVA084700, Merck Millipore Co., Billerica, MA, USA). From the results of quantification of proteins and carbohydrates (see section 2.6), almost all proteins were rejected by the PVC membrane, while the changes in carbohydrate concentration caused by filtration through the PVC membrane were negligible. By changing the mixing ratio of supernatant from which proteins were removed and untreated supernatant (protein concentration of approximately $60 \mathrm{mg} / \mathrm{L}$ ), four samples with different protein concentrations $(0,20,40$, and $60 \mathrm{mg} / \mathrm{L})$ were prepared. All samples had the same carbohydrate concentration of approximately 140 $\mathrm{mg} / \mathrm{L}$. The development of membrane fouling caused by these supernatants was evaluated by batch filtration tests using the micro-membrane modules described above.

\subsection{Evaluation of attachment of microbial flocs onto membrane surface}

To evaluate the propensity of microbial flocs to attach onto the surface of each 
membrane, a batch attachment test was carried out. Thin films (thickness: $500 \mu \mathrm{m}$ ) without any pores were prepared by casting and drying the solutions of the polymers used for membrane preparation in this study (i.e., PVDF, PVB, and CAB) with an applicator. These films were soaked in $10 \mathrm{~mL}$ of mixed liquor suspension for $1 \mathrm{~h}$, and then rinsed with pre-sterilized $0.85 \mathrm{wt} \% \mathrm{NaCl}$ aqueous solution to remove suspended solids accumulated on the film surface. After that, the bacteria and polysaccharides attached to the film surface were stained with a LIVE/DEAD BacLight Bacterial Viability Kit (Life Technologies, Carlsbad, CA, USA) and Concanavalin A conjugated with Alexa Fluor 647 (Con A, Life Technologies), respectively. Con A was used to visualize the $\alpha$-mannopyranosyl and $\alpha$-glucopyranosyl sugar residues contained in extracellular polymeric substances (EPS) [26, 27]. The stained films were then observed using a confocal laser scanning microscope (CLSM; FV1000D; Olympus, Tokyo, Japan) at $\mathrm{pH} 7.0$.

\subsection{Quantification of protein and carbohydrate concentration}

The bicinchoninic acid and phenol-sulfuric acid methods [28] were used to quantify protein and carbohydrate concentrations, respectively. Bovine serum albumin (BSA) and sodium alginate were used as standards for the quantitation of proteins and carbohydrates, respectively.

\section{Results and discussion}

3.1 Continuous operation of a laboratory-scale MBR equipped with membranes with different pore sizes 
Fig. 2 shows the changes in transmembrane pressure (TMPs) determined for three PVDF membranes with different pore sizes in Run 1. The rate of increase in TMP was in the following order (with respect to the pore size of the membranes): $0.02 \mu \mathrm{m}>$ $0.25 \mu \mathrm{m}>0.4 \mu \mathrm{m}$. This indicates that, at least for the PVDF membrane used in this study, membranes with smaller pores fouled more severely than those with larger ones. This trend was not in agreement with the results reported by Jin et al. [20]. In their study, a ceramic membrane with a pore size of $300 \mathrm{~nm}$ was fouled more severely than that with a pore size of $80 \mathrm{~nm}$. This discrepancy suggests that the development of membrane fouling cannot be explained solely by membrane pore size. Intrinsic membrane properties play important roles in determining the effect of membrane pore size on the development of membrane fouling. To optimize the design of membranes, comprehensive investigation of the optimum membrane pore size using membranes made of different polymeric materials is thought to be particularly useful. This will be discussed in the following sections.

\subsection{Batch filtration tests using micro-membrane modules}

The effect of membrane pore size on the development of membrane fouling was investigated using membranes made of three different polymeric materials, PVDF, $\mathrm{PVB}$, and $\mathrm{CAB}$. Taking the variability of the characteristics of mixed liquor suspensions of MBRs into consideration, the membranes to be compared should be examined simultaneously or at least during a limited time period without any large changes in mixed liquor characteristics. To acceralate the batch filtration tests, we carried out them in Run 2, which is more acceptable condition for bacterial growth and more severe condition for membrane fouling. Similar conditions have been used by other researchers 
$[14,17]$

Fig. 3 shows changes in TMP determined for the three PVDF membranes with different pore sizes in the batch filtration tests. The development of membrane fouling in the PVDF membranes with different pore sizes occurred in the descending order of $0.02 \mu \mathrm{m}>0.25 \mu \mathrm{m}>0.4 \mu \mathrm{m}$. The order of development of membrane fouling evaluated in the batch filtration test followed the order of the degree of membrane fouling developed in the continuous operation of the laboratory-scale MBR (Fig. 2). This suggests that the fouling propensity of each membrane on the laboratory-scale MBR can be reasonably estimated by the batch filtration test conducted in this study. In addition, the change in the characteristics of the mixed liquor suspension caused by the difference in the characteristics of the feed water between Runs 1 and 2 (see section 2.3) was not likely to be important. On this basis, the fouling propensities of the rest of the membranes prepared in this study were evaluated only by batch filtration tests.

\subsection{Effects of membrane pore size on the development of membrane fouling in} membranes made of different polymeric materials

Fig. 4 shows the relationship between membrane pore size and fouling rate determined in the batch filtration test. In this figure, the fouling rates are expressed as the time required until the TMP reached $30 \mathrm{kPa}$. A larger value in this index indicates that the development of membrane fouling in the corresponding filtration less occurred. In addition to membrane pore size, the fouling propensities of the prepared membranes were greatly affected by the membrane polymeric materials. When comparing the membrane fouling developed in the three membranes with small pore sizes (e.g., 0.02 $0.04 \mu \mathrm{m})$, membrane fouling occurred in descending order of membranes made of CAB 
> PVDF > PVB. Their water contact angles, which indicate the hydrophobicity of a material (higher water contact angle corresponds to a more hydrophobic nature), were in the following order: $\operatorname{PVDF}\left(84^{\circ}\right)>\operatorname{PVB}\left(77^{\circ}\right)>\mathrm{CAB}\left(75^{\circ}\right)$, indicating that PVDF is the most hydrophobic material used in this study. The discrepancy in the order of fouling rates determined in the batch filtration tests with the order of hydrophobicity of membrane polymeric materials suggests that the hydrophobicity of membrane polymeric materials is not a major factor determining the fouling propensity of a membrane, which is in agreement with some recent findings [18,19,21].

Fig. 4 indicates that the effect of membrane pore size on the development of membrane fouling differed considerably depending on the membrane polymeric material. As discussed previously, membrane fouling became less marked as membrane pore size became larger in PVDF membranes. In contrast, the opposite trend (i.e., membranes with larger pore size were more susceptible to membrane fouling) was seen for the $\mathrm{CAB}$ membranes. With regard to the membranes made of PVB, the effect of membrane pore size on the development of membrane fouling was negligible. Thus, it can be concluded that the effect of the membrane pore size and membrane material hydrophilicity should be considred together to have more realistic undrestanding of the fouling phenomena at MBR process. As far as we know, this is the first work that pore size dependence on the fouling are strongly affected by polymeric materials of used membranes.

\subsection{Major foulants of PVDF and CAB membranes}

Because trends in development of fouling in membranes with different pore sizes were opposite for membranes made of PVDF and CAB, the major foulants in 
these two membranes were investigated in detail. First, to investigate the main fraction in the mixed liquor suspension causing membrane fouling in these two membranes, batch filtration tests were conducted using two different feed suspensions: mixed liquor suspension without any treatment and the supernatant following centrifugation. The results of this experiment are shown in Fig. 5. Although the pore sizes of the membranes used in this experiment were almost similar, the trends in the development of membrane fouling were obviously different. In the PVDF membrane, the difference in the development of membrane fouling between the mixed liquor suspension and supernatant from centrifugation was small, suggesting that major foulants remained in the supernatant after centrifugation. In contrast, the development of membrane fouling in the $\mathrm{CAB}$ membrane was greatly suppressed after centrifugation. This suggests that microbial flocs removed by centrifugation are important components as foulants in CAB membranes.

To further confirm these observations, the constituents present in the supernatant following centrifugation that contributed to the development of membrane fouling in the PVDF membrane were investigated in detail. In many previous studies, proteins and polysaccharides were identified as major components in the foulants of MBRs. In this study, we investigated the influence of protein concentration on development of membrane fouling in the PVDF membrane by changing the protein concentration in the supernatant. As mentioned in Section 2.4, proteins contained in the supernatant can be removed selectively by filtering through a PVC membrane. Using this technique, supernatant samples with different protein concentrations and the same carbohydrate concentration were prepared. The profiles of TMP obtained in the batch filtration test using these supernatants are depicted in Fig. 6. The degree of membrane 
fouling developed in this batch filtration test was substantially suppressed as protein concentration decreased. In the filtered supernatant with a protein concentration of 0 $\mathrm{mg} / \mathrm{L}$, the increase in TMP during the batch filtration test was negligible. The carbohydrate concentrations of the samples used in this experiment were almost the same (approximately $140 \mathrm{mg} / \mathrm{L}$ ). The results obtained in this experiment strongly suggest that proteins played important roles in the development of membrane fouling in PVDF membranes due to the strong hydrophobic interaction between proteins and more hydrophobic PVDF. This is in agreement with recent findings [8,11,29-31].

To explain the difference in membrane fouling in each membrane caused by microbial flocs, the attachment propensity of a microbial suspension onto the surface of thin films made of each polymer was estimated by a batch attachment test. Fig. 7 shows the CLSM images of the film surfaces after the batch attachment test. On the surface of the thin films made of PVDF and PVB, the attachment of living bacteria was not significant. In contrast, attached bacteria were clearly visualized on the CAB film surface. The CLMS images shown in Fig. 7 (d), (e), and (f) also revealed the presence of more Con A on the CAB membrane than the PVDF and PVB membranes. The locations of the fluorescence emitted from Con A were almost the same as those of the attached microbial flocs, indicating that the microbial flocs attached to the film were accompanied with polysaccharides that can bind to Con A. The presence of Con A in CLMS images may indicate the location of EPS. These results indicate that the CAB film has a higher affinity for microbial flocs with EPS than the other membrane polymeric materials used in this study. These results readily explain the marked suppression of membrane fouling observed after removing microbial flocs by 
centrifugation (see Fig. 5(b)). However, the reason why microbial flocs are more likely to deposit on the CAB membrane is not clear. Further study is necessary to clarify this.

The results obtained in this study suggest that dominant fouling mechanisms differ depending on the membrane polymeric materials. In PVDF membranes, in which proteins are thought to be dominant foulants, inner-pore fouling caused by pore blocking or adsorption of foulants into the pore structure is likely to be the dominant fouling mechanism because protein size are small. In this case, fouling is more likely to occur for the membrane with smaller pore as shown in Fig. 4 because the pore blocking easily occures. Thus, optimization of structures inside the pores to improve water permeability (i.e., increasing the pathways by which water can pass through the membrane) would help to decrease the fouling propensity of PVDF membranes. In contrast, cake or gel layer formation of microbial flocs on the membrane surface is supposed to be the cause flocs are large and cannot go through the pores. Although the cake layer formation on the membrane surface is not so much different for membranes with different pore size, fouling is more likely to occur for the membrane with large pore as shown in Fig.4 because flocs can be partially penetrate inside the large pore. Because this type of cake laye formation strongly depends on the surface morphology such as roughness of the membrane $[20,21]$, control of surface morphology would be an effective countermeasure to mitigate membrane fouling in membranes with almost similar properties to the $\mathrm{CAB}$ membranes used in this study.

The information about the dominant foulant depending on the membrane polymeric materials is also practically useful. On the basis of the above discussion, the selection of operating conditions of MBRs should be changed depending on the type of membrane polymeric materials. For instance, when a membrane with almost similar 
properties to the PVDF membranes is employed, attention should be paid to controlling the concentration of organic compounds in which proteins are abundant. Because the contribution of such organic matter to membrane fouling is likely to be lowered by operating an MBR with relatively long SRT (and consequently low F/M ratio) $[11,15]$, it is suggested that operating an MBR with long SRT is particularly suitable for MBRs equipped with PVDF membranes. In contrast, such operating conditions are not recommended for membranes with almost similar properties to those of the $\mathrm{CAB}$ membranes, because of the high MLSS concentration caused by a long SRT. If the constructor of a treatment plant wants to take advantage of operating an MBR with short SRT or low MLSS concentration (e.g., improved oxygen transfer efficiency), a membrane with almost similar properties to the $\mathrm{CAB}$ membranes should be selected.

\section{Conclusions}

In this study, we investigated the relationship between membrane pore size and the development of membrane fouling. We used membranes made of three different polymeric materials, $\mathrm{PVDF}, \mathrm{PVB}$, and $\mathrm{CAB}$, to evaluate whether the membrane polymeric material affects this relationship. The results revealed that the relationship between membrane pore size and development of membrane fouling differed depending on the membrane polymeric material. For PVDF membranes, larger pores helped to mitigate the development of membrane fouling. In contrast, for CAB membranes, membranes with smaller pores had lower fouling propensity. This difference can be attributed to the different dominant foulants in each membrane. While microbial flocs are the dominant components of the foulants that caused membrane fouling in the $\mathrm{CAB}$ membranes, the contribution from organic compounds remaining in the supernatant 
following centrifugation were likely to predominate in membrane fouling in the PVDF membranes. On the basis of the results obtained in this study, it can be concluded that approaches by which the fouling propensities of membranes are minimized differed markedly depending on the type of membrane polymeric materials. Operating conditions of MBR should also be chosen by considering the characteristics of the membrane polymeric materials to be used.

\section{Acknowledgments}

This work was supported, in part, by the Regional Innovation Strategy Support Program, Ministry of Education, Culture, Sports, Science and Technology, Japan.

\section{References}

[1] T. Stephenson, S. Judd, B. Jefferson, K. Brindle, Membrane bioreactors for wastewater treatment, IWA publishing, London, 2000.

[2] S. Judd, The MBR Book, Elsevier, Amsterdam, 2006.

[3] M. Kraume, A. Drews, Membrane Bioreactors in Waste Water Treatment - Status and Trends, Chem Eng Technol, 33 (2010) 1251-1259.

[4] F. Meng, S.R. Chae, A. Drews, M. Kraume, H.S. Shin, F. Yang, Recent advances in membrane bioreactors (MBRs): membrane fouling and membrane material, Water Res, 43 (2009) 1489-1512.

[5] A. Drews, Membrane fouling in membrane bioreactors-Characterisation, contradictions, cause and cures, J Membrane Sci, 363 (2010) 1-28.

[6] G. Guglielmi, D. Chiarani, S.J. Judd, G. Andreottola, Flux criticality and sustainability in a hollow fibre submerged membrane bioreactor for municipal 
wastewater treatment, J Membrane Sci, 289 (2007) 241-248.

[7] G. Guglielmi, D. Chiarani, D.P. Saroj, G. Andreottola, Impact of chemical cleaning and air-sparging on the critical and sustainable flux in a flat sheet membrane bioreactor for municipal wastewater treatment, Water Sci Technol, 57 (2008) 1873-1879.

[8] K. Kimura, T. Miyoshi, T. Naruse, N. Yamato, R. Ogyu, Y. Watanabe, The difference in characteristics of foulants in submerged MBRs caused by the difference in the membrane flux, Desalination, 231 (2008) 268-275.

[9] Z. Wang, Z. Wu, G. Yu, J. Liu, Z. Zhou, Relationship between sludge characteristics and membrane flux determination in submerged membrane bioreactors, J Membrane Sci, 284 (2006) 87-94.

[10] M.A.H. Johir, J. George, S. Vigneswaran, J. Kandasamy, A. Sathasivan, A. Grasmick, Effect of imposed flux on fouling behavior in high rate membrane bioreactor, Biores Technol, 122 (2012) 42-49.

[11] T. Miyoshi, T. Tsuyuhara, R. Ogyu, K. Kimura, Y. Watanabe, Seasonal variation in membrane fouling in membrane bioreactors (MBRs) treating municipal wastewater, Water Res, 43 (2009) 5109-5118.

[12] R. van den Broeck, J. van Dierdonck, P. Nijskens, C. Dotremont, P. Krzeminski, J.H.J.M. van der Graaf, J.B. van Lier, J.F.M. van Impe, I.Y. Smets, The influence of solids retention time on activated sludge bioflocculation and membrane fouling in a membrane bioreactor (MBR), J Membrane Sci, 401-402 (2012) 48-55.

[13] G. Sabia, M. Ferraris, A. Spagni, Effect of solid retention time on sludge filterability and biomass activity: Long-term experiment on a pilot-scale membrane bioreactor treating municipal wastewater, Chem Eng J, 221 (2013) 176-184.

[14] C. Huyskens, S. Lenaerts, E. Brauns, L. Diels, H. De Wever, Study of (ir)reversible 
fouling in MBRs under various operating conditions using new on-line fouling sensor, Sep Purif Technol, 81 (2011) 208-215.

[15] K. Kimura, N. Yamato, H. Yamamura, Y. Watanabe, Membrane fouling in pilot-scale membrane Bioreactors (MBRs) treating municipal wastewater, Environ Sci Technol, 39 (2005) 6293-6299.

[16] R.S. Trussell, R.P. Merlo, S.W. Hermanowicz, D. Jenkins, The effect of organic loading on process performance and membrane fouling in a submerged membrane bioreactor treating municipal wastewater, Water Res, 40 (2006) 2675-2683.

[17] N. Yamato, K. Kimura, T. Miyoshi, Y. Watanabe, Difference in membrane fouling in membrane bioreactors (MBRs) caused by membrane polymer materials, J Membrane Sci, 280 (2006) 911-919.

[18] J.H. Choi, H.Y. Ng, Effect of membrane type and material on performance of a submerged membrane bioreactor, Chemosphere, 71 (2008) 853-859.

[19] J.H. Choi, S.-K. Park, H.-Y. Ng, Membrane fouling in a submerged membrane bioreactor using track-etched and phase-inversed porous membranes, Sep Purif Technol, 65 (2009) 184-192.

[20] L. Jin, S.L. Ong, H.Y. Ng, Comparison of fouling characteristics in different pore-sized submerged ceramic membrane bioreactors, Water Res, 44 (2010) 5907-5918. [21] T. Tsuyuhara, Y. Hanamoto, T. Miyoshi, K. Kimura, Y. Watanabe, Influence of membrane properties on physically reversible and irreversible fouling in membrane bioreactors, Water Sci Technol, 61 (2010) 2235-2240.

[22] P. van der Marel, A. Zwijnenburg, A. Kemperman, M. Wessling, H. Temmink, W. van der Meer, Influence of membrane properties on fouling in submerged membrane bioreactors, J Membrane Sci, 348 (2010) 66-74. 
[23] H. Matsuyama, H. Okafuji, T. Maki, M. Teramoto, N. Kubota, Preparation of polyethylene hollow fiber membrane via thermally induced phase separation, J Membrane Sci, 223 (2003) 119-126.

[24] M. Hashino, T. Katagiri, N. Kubota, Y. Ohmukai, T. Maruyama, H. Matsuyama, Effect of membrane surface morphology on membrane fouling with sodium alginate, $\mathbf{J}$ Membrane Sci, 366 (2011) 258-265.

[25] T. Kitaura, W.N. Fadzlina, Y. Ohmukai, T. Maruyama, H. Matsuyama, Preparation and characterization of several types of polyvinyl butyral hollow fiber membranes by thermally induced phase separation, J Appl Polym Sci, 127 (2013) 4072-4078.

[26] T.C.A. Ng, H.Y. Ng, Characterisation of initial fouling in aerobic submerged membrane bioreactors in relation to physico-chemical characteristics under different flux conditions, Water Res, 44 (2010) 2336-2348.

[27] O. Nosyk, E. ter Haseborg, U. Metzger, F.H. Frimmel, A standardized pre-treatment method of biofilm flocs for fluorescence microscopic characterization, $\mathrm{J}$ Microbiol Methods, 75 (2008) 449-456.

[28] M. Dubois, K.A. Gilles, J.K. Hamilton, P.A. Rebers, F. Smith, Colorimetric method for determination of sugars and related substances, Anal Chem, 28 (1956) 350-356.

[29] S. Tang, Z. Wang, Z. Wu, Q. Zhou, Role of dissolved organic matters (DOM) in membrane fouling of membrane bioreactors for municipal wastewater treatment, $\mathrm{J}$ Hazard Mater, 178 (2010) 377-384.

[30] Y. Tian, L. Chen, S. Zhang, S. Zhang, A systematic study of soluble microbial products and their fouling impacts in membrane bioreactors, Chem Eng J, 168 (2011) 1093-1102.

[31] T. Miyoshi, T. Aizawa, K. Kimura, Y. Watanabe, Identification of proteins involved 
in membrane fouling in membrane bioreactors (MBRs) treating municipal wastewater, Int Biodeter Biodegr, 75 (2012) 15-22. 
Fig. 1. Schematic diagram of the experimental apparatus used in batch filtration tests.

Fig. 2. Changes in TMP during continuous operation of a laboratory-scale MBR equipped with PVDF membranes with different pore sizes.

Fig. 3. Changes in TMP during a batch filtration test using mini-modules made of PVDF membranes with different pore sizes.

Fig. 4. Relationship between membrane pore size and fouling rate determined in a batch filtration test. Squares: PVDF membranes, circles: CAB membranes, Triangle: PVB membranes.

Fig. 5. Changes in TMP during batch filtration tests using mini-modules. TMPs recorded in filtration of mixed liquor suspension $(\square)$ and supernatant $(O)$ from centrifugation. The pore sizes of PVDF and CAB membranes were 0.25 and $0.2 \mu \mathrm{m}$, respectively.

Fig. 6. Changes in TMP during a batch filtration test using a PVDF membrane with different protein concentrations. Open squares: $0 \mathrm{mg} / \mathrm{L}$, circles: $20 \mathrm{mg} / \mathrm{L}$, open circle: $40 \mathrm{mg} / \mathrm{L}$, triangles: $60 \mathrm{mg} / \mathrm{L}$. All samples had the same carbohydrate concentration of approximately $140 \mathrm{mg} / \mathrm{L}$.

Fig. 7. CLSM images obtained for the surface of thin films used in a batch attachment test with mixed liquor suspension. The surfaces of the thin films were stained with (a)-(c) LIVE/DEAD stain (Molecular Probes Inc.) and (D)-(F) Con A conjugated with Alexa Fluor 647. (a) and (d) were PVDF films, (b) and (e) were PVB films, and (c) and (f) were CAB films. Scale bars in panels (a-f) are $20 \mu \mathrm{m}$. 


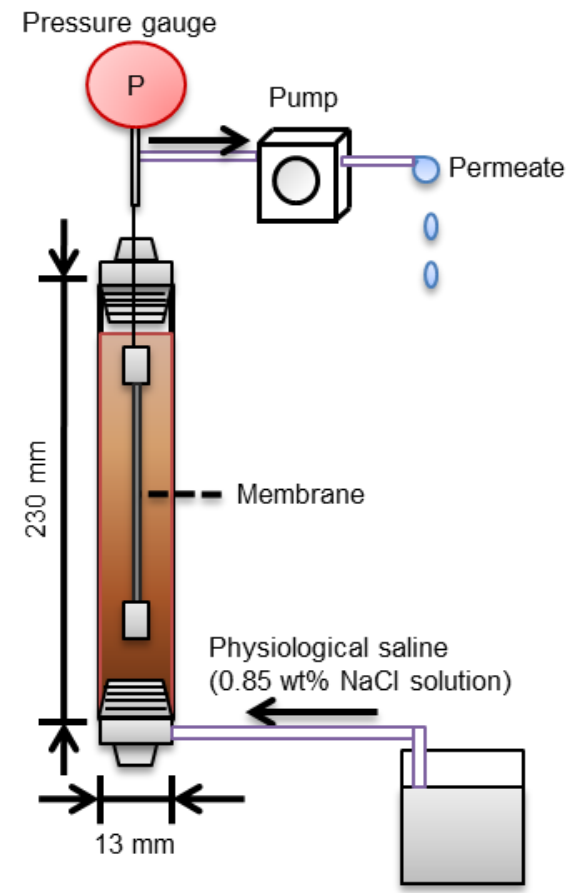

Fig. 1. Schematic diagram of the experimental apparatus used in batch filtration tests.

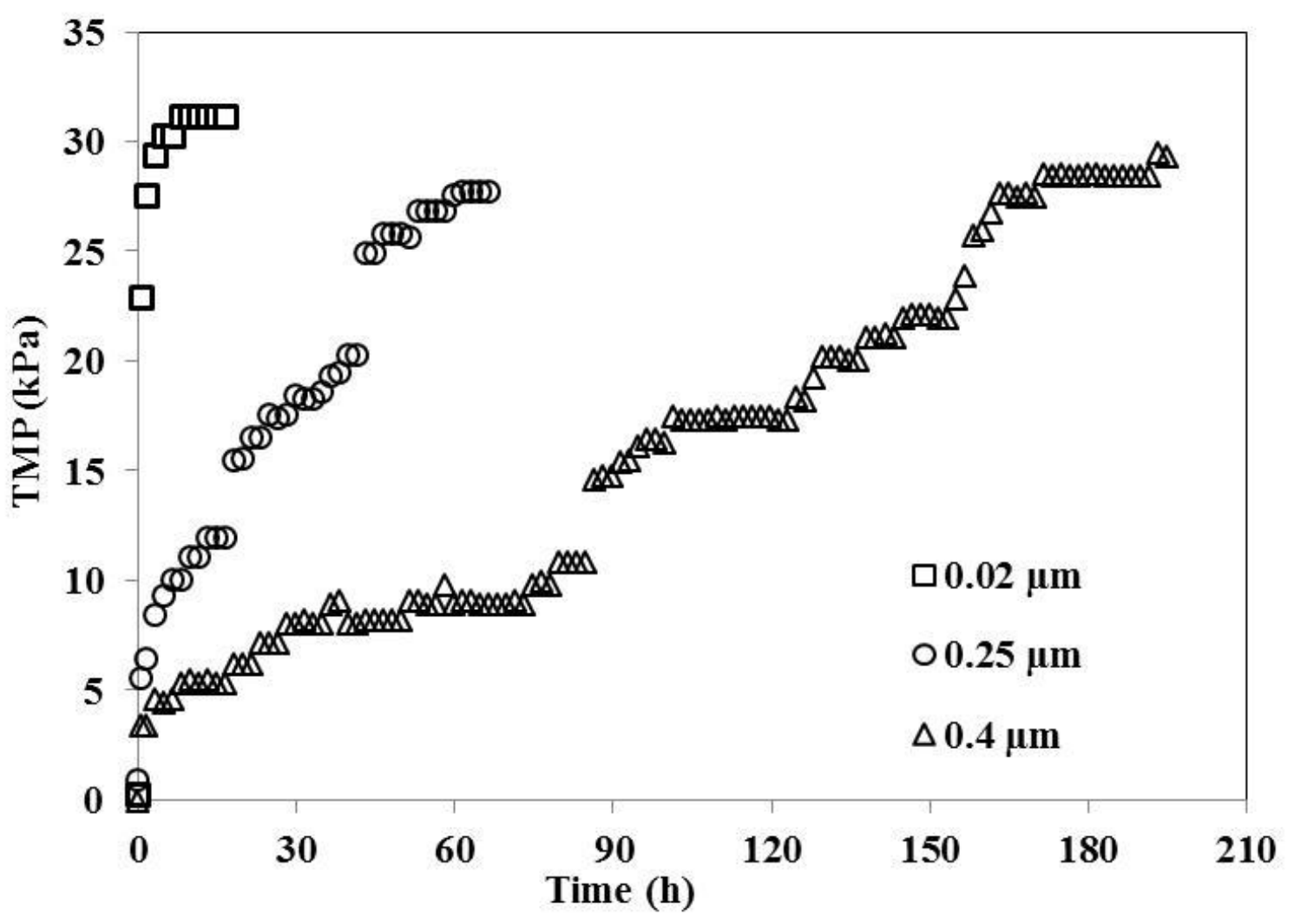

Fig. 2. Changes in TMP during continuous operation of a laboratory-scale MBR equipped with PVDF membranes with different pore sizes. 


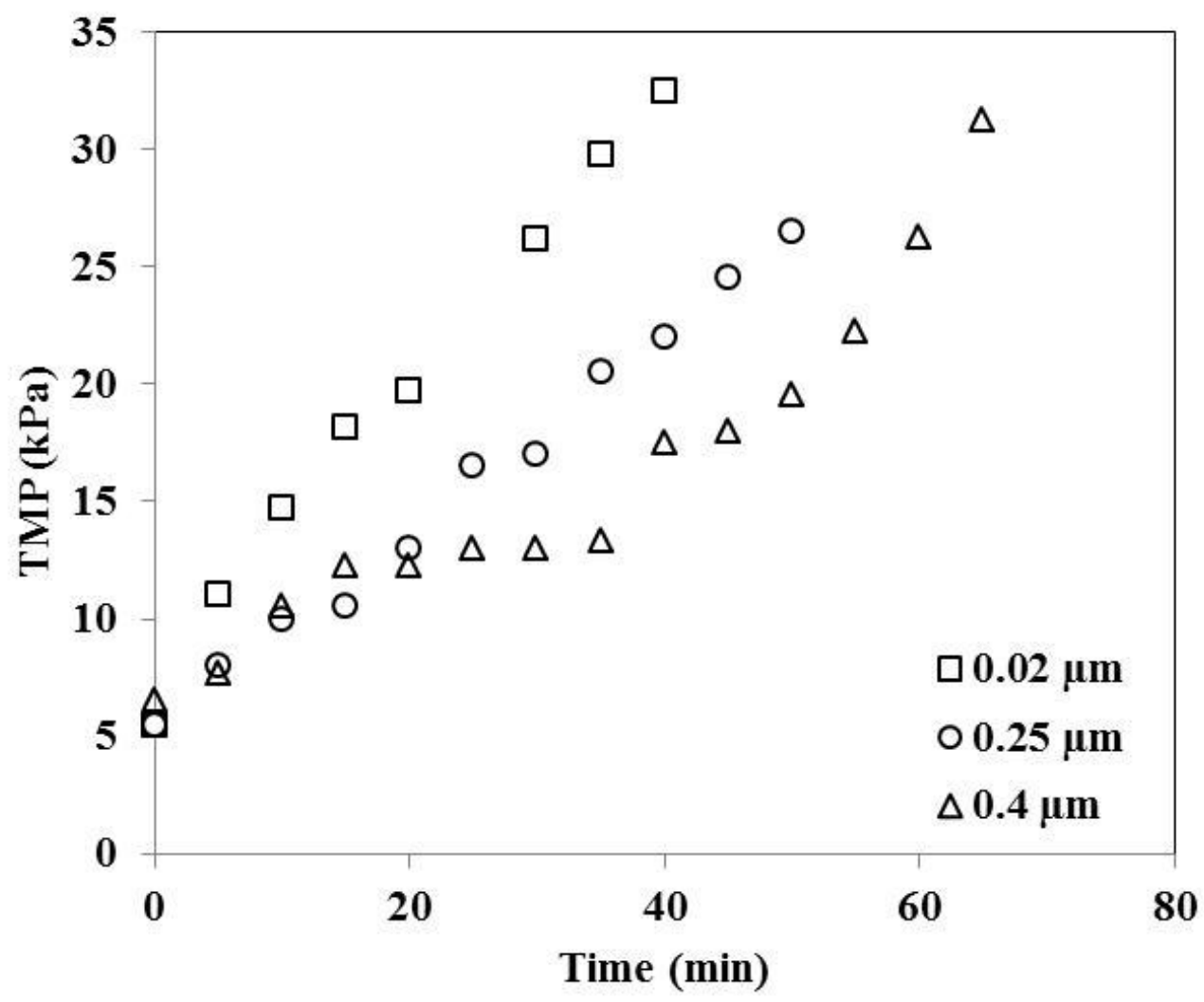

Fig. 3. Changes in TMP during a batch filtration test using mini-modules made of PVDF membranes with different pore sizes.

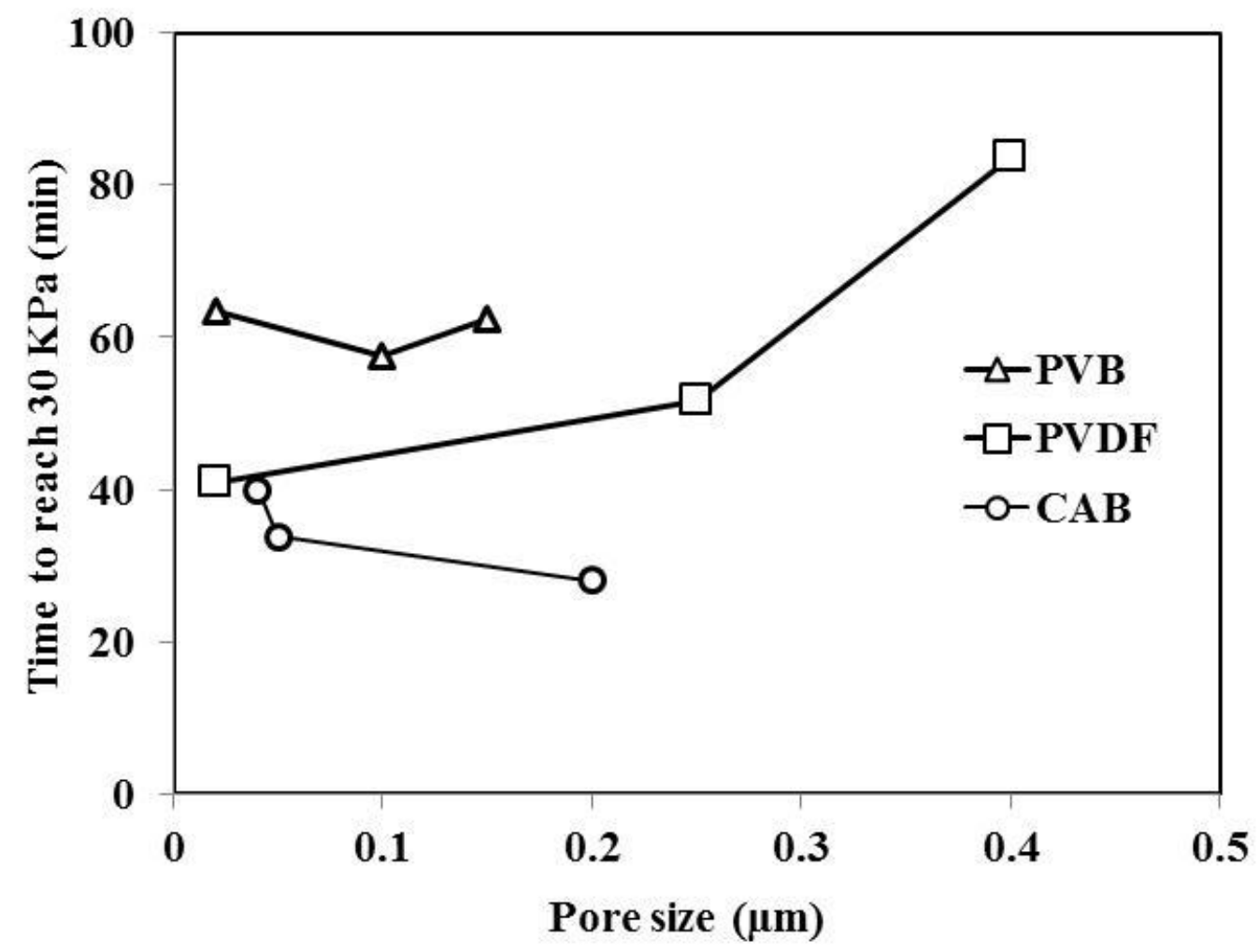

Fig. 4. Relationship between membrane pore size and fouling rate determined in a batch filtration test. Squares: PVDF membranes, circles: CAB membranes, Triangle: PVB membranes. 

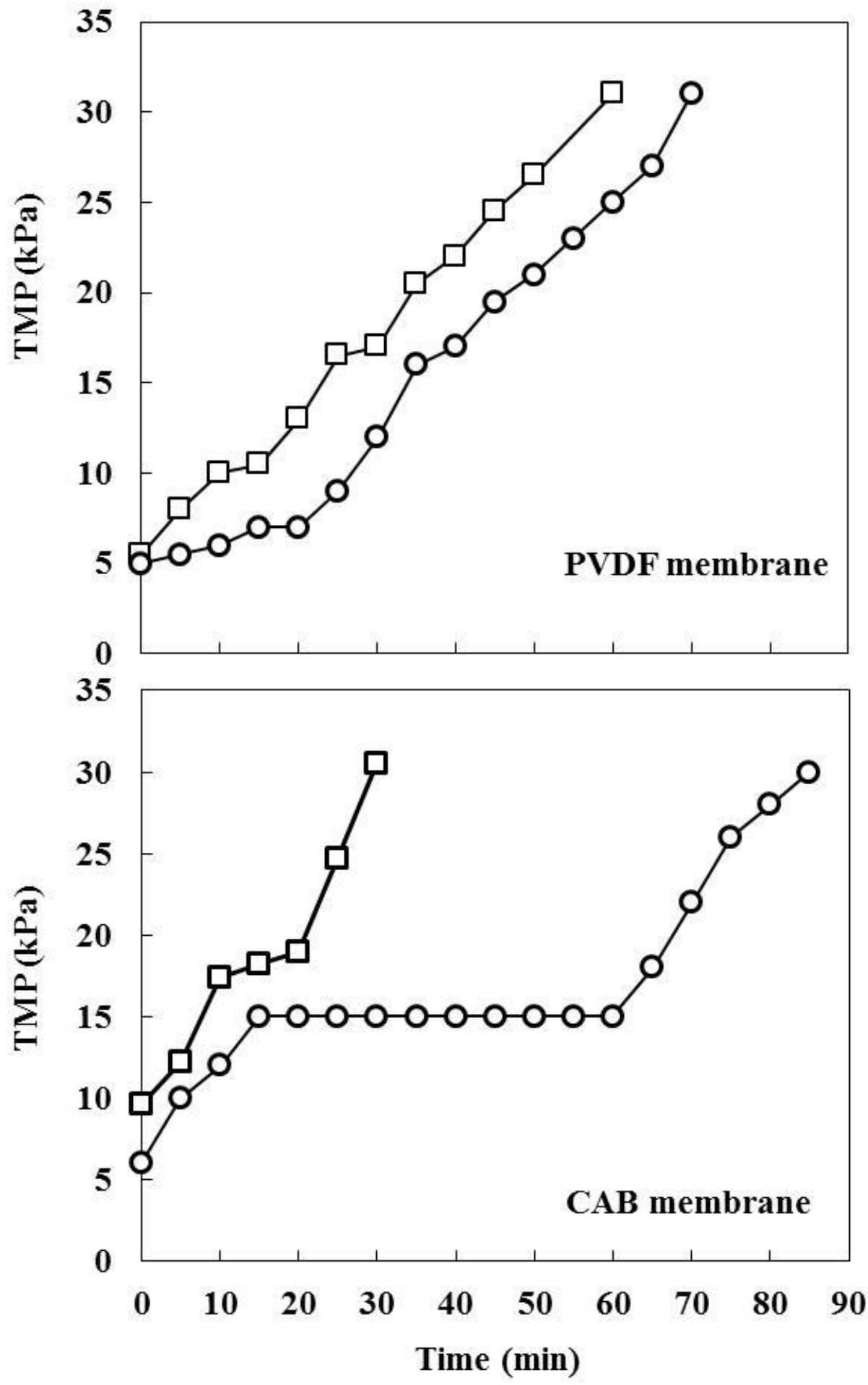

Fig. 5. Changes in TMP during batch filtration tests using mini-modules. TMPs recorded in filtration of mixed liquor suspension $(\square)$ and supernatant $(O)$ from centrifugation. The pore sizes of PVDF and CAB membranes were 0.25 and $0.2 \mu \mathrm{m}$, respectively. 


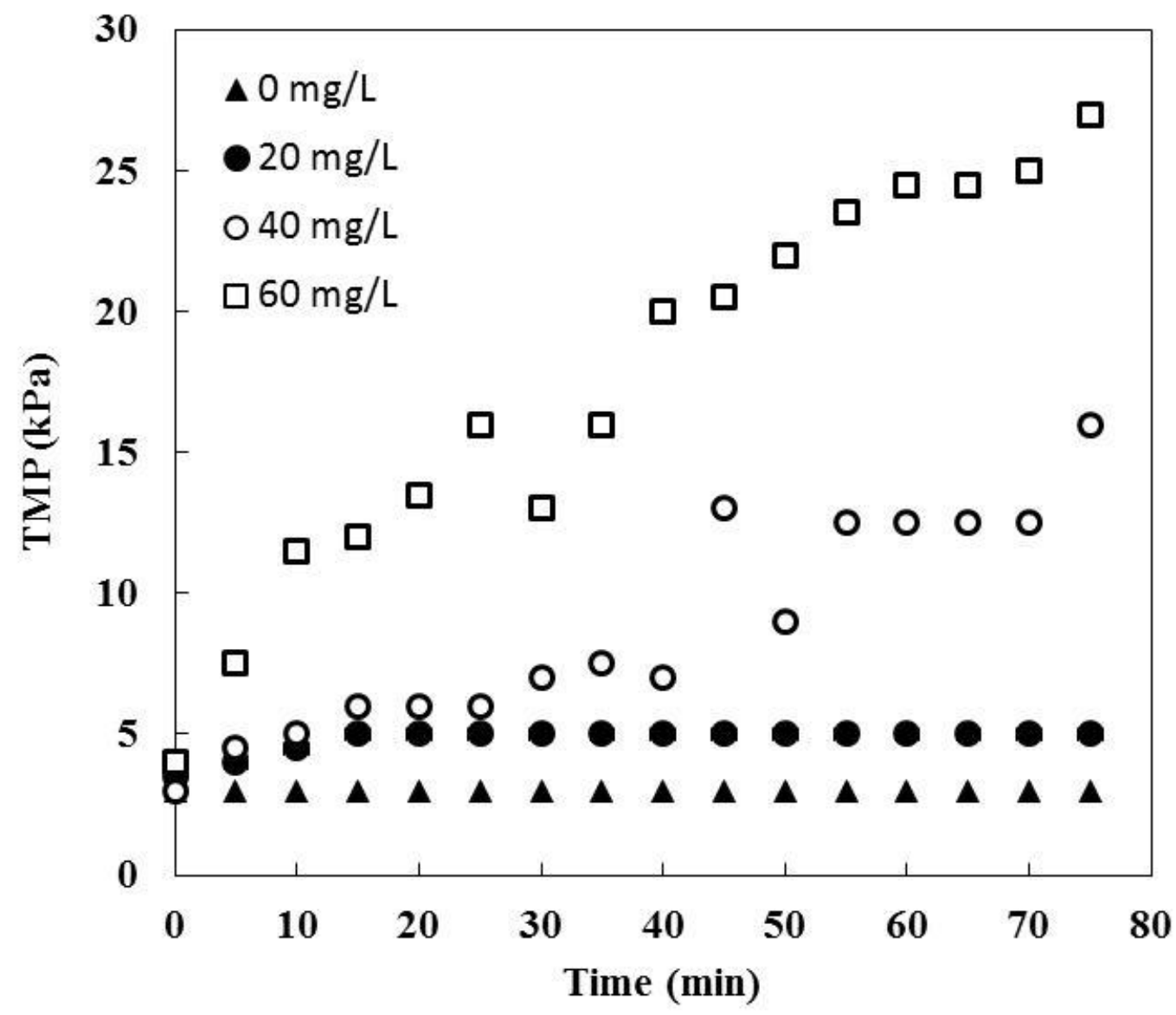

Fig. 6. Changes in TMP during a batch filtration test using a PVDF membrane with different protein concentrations. Open squares: $0 \mathrm{mg} / \mathrm{L}$, circles: $20 \mathrm{mg} / \mathrm{L}$, open circle: $40 \mathrm{mg} / \mathrm{L}$, triangles: $60 \mathrm{mg} / \mathrm{L}$. All samples had the same carbohydrate concentration of approximately $140 \mathrm{mg} / \mathrm{L}$. 

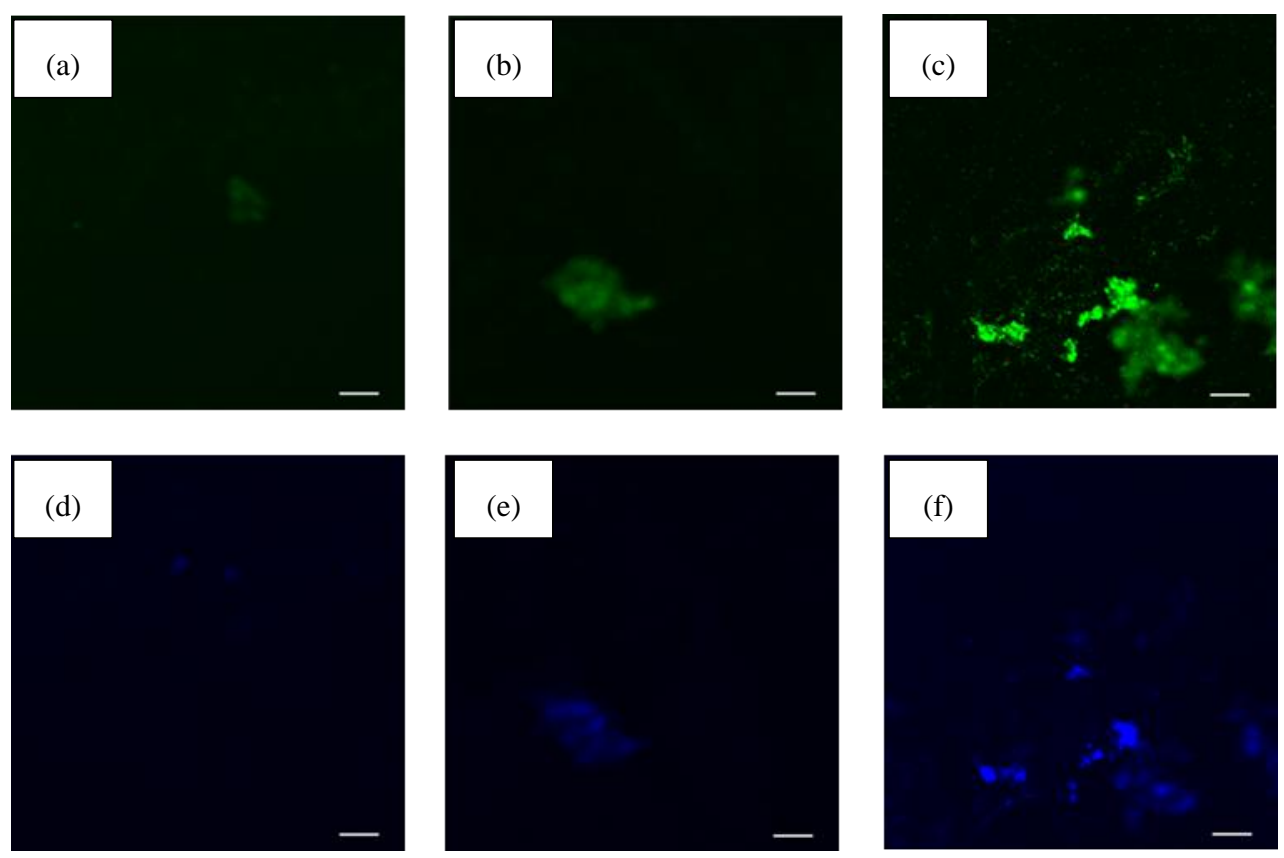

Fig. 7. CLSM images obtained for the surface of thin films used in a batch attachment test with mixed liquor suspension. The surfaces of the thin films were stained with (a)-(c) LIVE/DEAD stain (Molecular Probes Inc.) and (D)-(F) Con A conjugated with Alexa Fluor 647. (a) and (d) were PVDF films, (b) and (e) were PVB films, and (c) and (f) were CAB films. Scale bars in panels (a-f) are $20 \mu \mathrm{m}$. 


\section{Table 1}

Preparation conditions of hollow fiber membranes.

\begin{tabular}{|c|c|c|c|c|c|c|c|c|c|}
\hline & \multicolumn{3}{|c|}{$\mathrm{CAB}$} & \multicolumn{3}{|c|}{ PVB } & \multicolumn{3}{|c|}{ PVDF } \\
\hline & $0.2 \mu \mathrm{m}$ & $0.05 \mu \mathrm{m}$ & $0.04 \mu \mathrm{m}$ & $0.15 \mu \mathrm{m}$ & $0.1 \mu \mathrm{m}$ & $0.02 \mu \mathrm{m}$ & $0.4 \mu \mathrm{m}$ & $0.25 \mu \mathrm{m}$ & $0.02 \mu \mathrm{m}$ \\
\hline Polymer solution & \multicolumn{3}{|c|}{ CAB-TEG } & \multicolumn{3}{|c|}{ PVB-PEG } & \multicolumn{3}{|c|}{ PVDF-DEP } \\
\hline Polymer concentration & $12 \mathrm{wt} \%$ & $15 \mathrm{wt} \%$ & $20 \mathrm{wt} \%$ & $15 \mathrm{wt} \%$ & $18 \mathrm{wt} \%$ & $20 \mathrm{wt} \%$ & $25 \mathrm{wt} \%$ & $28 \mathrm{wt} \%$ & $35 \mathrm{wt} \%$ \\
\hline Solution temperature $(\mathrm{K})$ & & 443 & & & 443 & & & 463 & \\
\hline Flow rate of polymeric solution $(\mathrm{g} / \mathrm{s})$ & 0.65 & 0.45 & 0.44 & 0.22 & 0.18 & 0.17 & 0.18 & 0.15 & 0.13 \\
\hline Flow rate of diluent $(\mathrm{g} / \mathrm{s})$ & 0.10 & 0.10 & 0.088 & 0.10 & 0.10 & 0.097 & 0.097 & 0.10 & 0.10 \\
\hline Take-up speed (m/s) & 0.39 & 0.37 & 0.37 & 0.26 & 0.26 & 0.24 & 0.11 & 0.14 & 0.14 \\
\hline Air gap (mm) & \multicolumn{3}{|c|}{2} & \multicolumn{3}{|c|}{0} & \multicolumn{3}{|c|}{5} \\
\hline Batch composition & \multicolumn{3}{|c|}{ water } & \multicolumn{3}{|c|}{ water } & \multicolumn{3}{|c|}{ water } \\
\hline Batch temperature $(\mathrm{K})$ & \multicolumn{3}{|c|}{313} & \multicolumn{3}{|c|}{313} & \multicolumn{3}{|c|}{273} \\
\hline
\end{tabular}

